\title{
Evaluation of carbon nanotubes as a solid-phase extraction adsorbent for the extraction of cephalosporins antibiotics, sulfonamides and phenolic compounds from aqueous solution
}

\author{
Hongyun $\mathrm{Niu}^{\mathrm{a}}$, Yaqi Cai ${ }^{\mathrm{a}, *}$, Yali Shi ${ }^{\mathrm{a}}$, Fusheng $\mathrm{Wei}^{\mathrm{b}, * *}$, \\ Jiemin Liu ${ }^{\mathrm{c}}$, Shifen Mou ${ }^{\mathrm{a}}$, Guibin Jiang ${ }^{\mathrm{a}}$ \\ ${ }^{a}$ State Key Laboratory of Environmental Chemistry and Ecotoxicology, Research Center for Eco-Environmental Science, \\ Chinese Academy of Sciences, Beijing 100085, China \\ ${ }^{\mathrm{b}}$ China National Environmental Monitoring Center, Beijing 100029, China \\ ${ }^{c}$ Department of Chemistry, University of Science and Technology Beijing, Beijing 100083, China \\ Received 9 February 2007; received in revised form 11 May 2007; accepted 11 May 2007 \\ Available online 21 May 2007
}

\begin{abstract}
The adsorptive potential of carbon nanotubes (single-walled carbon nanotubes and multi-walled carbon nanotubes) for solid-phase extraction of three groups of highly polar compounds (namely cephalosporins antibiotics, sulfonamides and phenolic compounds) was tested in this article. The analytes were strongly retained by the carbon nanotubes. And acceptable recoveries were obtained with the addition of ammonium acetate into eluents. The effects of solution $\mathrm{pH}$ on the recoveries of the antibiotics and phenolic compounds were examined. To check the retention abilities of three groups of compounds on carbon nanotubes, fixed amount of each analyte was added to different volumes (up to $500 \mathrm{~mL}$ ) of aqueous solution, and then extracted by the sorbents. Comparative studies showed that the carbon nanotubes were much superior to $\mathrm{C}_{18}$ for the extraction of the highly polar analytes. For the cephalosporins antibiotics and sulfonamides, the carbon nanotubes showed stronger retention capability than graphitized carbon blacks, but for some of the phenolic compounds graphitized carbon blacks seemed to be more suitable, indicating different retention mechanisms of these analytes. To further assess the enrichment ability of carbon nanotubes for highly polar compounds, the solid-phase extraction method of multi-walled carbon nanotubes packed cartridge was well developed, and the sulfonamides were used as model compounds. Under the optimal procedures, the detection limits of sulfonamides were in the range of $27-38 \mathrm{ng} \mathrm{L}^{-1}$. The spiked recoveries from several real water samples obtained for sulfathiazole and sulfadiazine ranged from $55 \%$ to $79 \%$ and $72 \%$ to $92 \%$, respectively, while the recoveries of sulfapyridine and sulfamethazine were in the range of $85-102 \%$.
\end{abstract}

(C) 2007 Elsevier B.V. All rights reserved.

Keywords: Carbon nanotubes; Solid-phase extraction; Highly polar compounds

\section{Introduction}

Solid-phase extraction (SPE) has become a well-established preconcentration technique in environmental analytical applications in recent years [1-5]. And a wide range of analytes including non-polar, moderately polar and polar ones present

\footnotetext{
* Corresponding author at: Chinese Academy of Sciences, Research Center for Eco-Environmental Science, P.O. Box 2871, Beijing 100085, China.

Tel.: +8610 62849182; fax: +861062849239.

** Corresponding author.

E-mail addresses: caiyaqi@ rcees.ac.cn (Y. Cai), weifs@public3.bta.net.cn (F. Wei).
}

in samples can be extracted by various sorbents. However, among all the organic compounds, polar compounds particularly have received most attention because of their low affinity for most reversed-phase sorbents (such as silica or polystyrenedivinylbenzene (PS-DVB) polymeric sorbents) [2-4]. New materials mainly polymeric sorbents have been developed in the last few years to improve their retention towards polar compounds [5,6]. For this kind of sorbents, there are two parameters, special surface area and hydrophilicity of the polymeric sorbents that can affect the adsorption of the polar compounds [4]. Some commercial available hypercrosslinked polystyrene sorbents such as Isolute ${ }^{\circledR}$ ENV+ (IST) and Lichrolut ${ }^{\circledR}$ EN (Merck) with increasing special surface area (over $1000 \mathrm{~m}^{2} \mathrm{~g}^{-1}$ ) can improve 
the retention of moderately polar compounds [4-6]. However, due to the hydrophobicity of those highly crosslinked sorbents, low recoveries are obtained when they are used to analyze highly polar analytes. $[4,6]$. To overcome the hydrophobicity of the resins, new hydrophilic polymeric materials have been developed, and Oasis hydrophilic-lipophilic balance (HLB) copolymer (Waters) is one of the hydrophilic sorbents recognized world-widely $[6,7]$.

Similar to the polymers materials, other materials such as carbon nanotubes have been the subject of the research to develop new SPE sorbents for polar compounds. Due to their excellent mechanical, chemical and electrical property, carbon nanotubes (CNTs) have attained considerable attention and have been used in many fields since their discovery in 1991 [8-10]. The structure of CNTs resembles one (single-walled carbon nanotubes, SWCNTs) or more graphite sheets (multiwalled carbon nanotubes, MWCNTs) rolled up into a cylinder that consists of hexagon-rich $\mathrm{sp}^{2}$ carbon at the wall and a few pentagons at the caps, getting more $\mathrm{sp}^{3}$-like as tube diameters become smaller $(<0.7 \mathrm{~nm})$ [11]. Because of the larger specific area and hydrophobic characteristic of the surface, CNTs have been regarded as a new kind of sorbent and have been studied for the removal of dioxin [12], trihalomethanes [13], microcystins [14], 1,2-dichlorobenzene [15], nicotine and tar [16]. They also showed satisfactory analytical potential as effective solid-phase extraction adsorbents for cyanazine [17], chlorotoluron [18], bisphenol A, 4-n-nonylphenol, 4-tert-octylphenol [19], chlorophenols [20], and several phthalate esters [21], non-steroidal anti-inflammatory drugs [22], and polybrominated diphenyl ethers [23]. And CNTs have been used as adsorbents for trapping or separation of volatile organic compounds, indicating the potential in chromatographic applications [11,24-26]. All these studies mentioned above reveal that CNTs are the promising materials in SPE fields. However, as to our knowledge, only a little kind of moderately polar or polar compounds, such as atrazine and simazine [27], sulfonylurea herbicides [28], organophosphorus pesticides [29], drugs of propranolol, cinchonine and quinine [30], and dicamba herbicide [31] have been concentrated by CNTs. Then the purpose of our experiment was to investigate the applicability of CNTs as packing adsorbents for solid-phase extraction of highly polar analytes.

In the present study, the SPE cartridges packed with SWCNTs and MWCNTs were self-made in our lab and two groups of antibiotics (cephalosporin antibiotics and sulfonamide antibiotics) and a group of phenolic compounds were extracted. The polar characteristic of the analytes was shown by their low octanol-water partition coefficient $\left(K_{\mathrm{ow}}\right)$. In log unit, the values for the antibiotics were less than 1 and for most of the phenolic compounds the values were less than 2 . For comparison, the retention abilities of the classical $\mathrm{C}_{18}$ silica sorbent and graphitized carbon blacks (GCBs) (an important carbonaceous sorbent) to the selected analytes were also investigated. Finally, the preconcentration method with MWCNTs packed cartridge based on the optimal condition was well developed and applied to the analysis of several environmental water samples. In this section, sulfonamides were used as model analytes.

\section{Experimental}

\subsection{Materials}

The selected compounds consisted of 6 phenolic compounds, 4 cephalosporin antibiotics and 4 sulfonamide antibiotics. Phenolic compounds was made up of phenol, benzoic acid, hydroxyquinone, supplied by Institute of Environmental protection and monitoring, Department of Agriculture (Beijing, China); and guaiacol, 1,3,5-trihydroxybenzene and 3,5-dihydroxybenzoic acid, obtained from Sigma-Aldrich (Steinheim, Germany). Cephalosporin antibiotics included cephalotin, cephalexin, cephradine and cephaclor, purchased from National Institute for the Control of Pharmaceutical and Biological Products (Beijing, China). And sulfonamide antibiotics comprised sulfathiazole, sulfadiazine, sulfapyridine, from Tokyo Kasei Kogyo Co. Ltd. (Tokyo, Japan) and sulfamethazine, from Acros (Morris Plains, New Jersey, USA). Chemical structures of the selected compounds were shown in Table 1. HPLC-grade acetonitrile (ACN) and methanol $(\mathrm{MeOH})$ were obtained from Merck (Darmstadt, Germany). Standard stock solutions $\left(400 \mu \mathrm{g} \mathrm{mL}^{-1}\right.$ or $200 \mu \mathrm{g} \mathrm{mL}^{-1}$ ) of each compound were prepared in methanol or purified water from Barnstead EASYpure ${ }^{\circledR}$ II LF system (Barnstead Int., Dubuque, IA, USA). The mixtures of each group of the compounds were prepared by diluting the standard solution with purified water. Sodium hydroxide and hydrochloric acid were guarantee grade reagents (Beijing Chemical Corporation, Beijing, China). Agilent ZORBAX SPE $\mathrm{C}_{18}$ silica sorbents were purchased from Agilent Technologies (Santa Clara, CA, USA). The Supelco ENVI-Carb tubes were purchased from Supelco (Bellefonte, PA, USA), and the diameters and specific surface areas of the GCBs sorbent were about $38-150 \mu \mathrm{m}, 100 \mathrm{~m}^{2} \mathrm{~g}^{-1}$, respectively. MWCNTs with an average external diameter of $30-60 \mathrm{~nm}$ and specific surface areas $131.74 \mathrm{~m}^{2} \mathrm{~g}^{-1}$ were provided by Tsinghua-Nafine Nano-Powder Commercialization Engineering Center, Chemical Engineering Department of Tsinghua University (Beijing, China). SWCNTs were kindly donated by Nankai University (Tianjin, China) and the diameters and specific surface areas of the SWCNTs sorbent were about $0.5-1.6 \mathrm{~nm}$ and $297.72 \mathrm{~m}^{2} \mathrm{~g}^{-1}$, respectively.

\subsection{Chromatographic conditions}

The chromatographic experiments were performed by using Dionex SUMMIT ${ }^{\circledR}$ high performance liquid chromatography (HPLC) system (Dionex, Sunyvale, CA, USA) included two P680 HPLC pumps, an on-line connected degasser Solvent Rack SOR-100, an ASI-100 automated sample injector, a thermostatted column compartment TCC-100, and a PDA-100 photodiode array detector. The analytical columns included a DIONEX Acclaim Polar Advantage $\mathrm{C}_{16}$ column $(150 \times 4.6 \mathrm{~mm}$; particle size, $5 \mu \mathrm{m}$ ) for the separations of cephalosporin antibiotics, and a DIONEX Acclaim Polar Advantage $\mathrm{C}_{18}$ column $(150 \times 2.1 \mathrm{~mm}$, particle size, $3 \mu \mathrm{m})$ for the other compounds. Gradient separations for cephalosporin antibiotics were carried out using phosphate buffer ( $\mathrm{pH} 3.0$, adjusted by $0.2 \mathrm{M}$ potas- 
Table 1

Structure, dissociation constants $\left(\mathrm{p} K_{\mathrm{a}}\right)$ and octanol/water partition constants $\left(\log K_{\mathrm{OW}}\right)$ of the analytes

\begin{tabular}{|c|c|c|c|}
\hline Name & Structure & $\mathrm{p} K \mathrm{a}^{\mathrm{a}}$ & $\log K_{\mathrm{OW}}$ \\
\hline Cephaclor & & $\begin{array}{l}\mathrm{p} K \mathrm{a}_{1}=1.5 \\
\mathrm{p} K \mathrm{a}_{2}=7.2\end{array}$ & $0.35^{\mathrm{a}}$ \\
\hline Cephalexin & & $\begin{array}{l}\mathrm{p} K \mathrm{a}_{1}=2.5 \\
\mathrm{p} K \mathrm{a}_{2}=5.2 \\
\mathrm{p} K \mathrm{a}_{3}=7.3\end{array}$ & $0.6^{\mathrm{a}}$ \\
\hline Cephradine & & $\begin{array}{l}\mathrm{p} K \mathrm{a}_{1}=2.5 \\
\mathrm{p} K \mathrm{a}_{2}=7.3\end{array}$ & $0.4^{\mathrm{a}}$ \\
\hline Cephalotin & & $\mathrm{p} K \mathrm{a}_{1}=2.2\left(35^{\circ} \mathrm{C}\right)$ & $\begin{array}{l}0.08^{\mathrm{b}}\left(35^{\circ} \mathrm{C}\right) \\
0^{\mathrm{a}}\end{array}$ \\
\hline
\end{tabular}

Name

Sulfonamide antibiotics

Sulfadiazine

Sulfathiazole

\section{Structure}
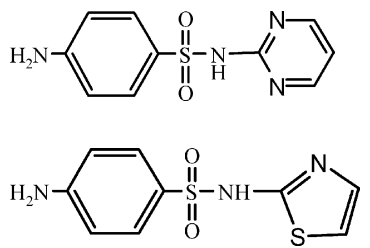

Sulfapyridine
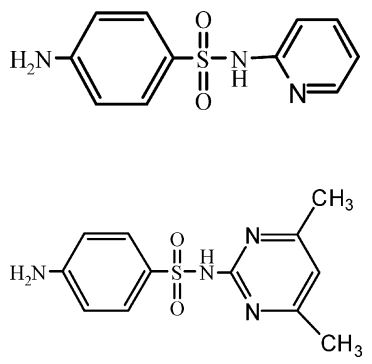

$\mathrm{p} K \mathrm{a}^{\mathrm{c}}$

$\mathrm{p} K \mathrm{a}_{1}=2.14$

$\mathrm{p} K \mathrm{a}_{2}=6.34$

$\mathrm{p} K \mathrm{a}_{1}=2.54$

$\mathrm{p} K \mathrm{a}_{2}=6.98$

$\mathrm{p} K \mathrm{a}_{1}=2.74$

$\mathrm{p} K \mathrm{a}_{2}=8.29$

$\mathrm{p} K \mathrm{a}_{1}=2.54$

$0.25^{\mathrm{d}}$

$\mathrm{p} K \mathrm{a}_{2}=7.22$

$\mathrm{p} K \mathrm{a}$

$\log K_{\text {OW }}$

Phenolic compounds

Phenol

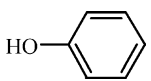

$\mathrm{p} K \mathrm{a}=9.89^{\mathrm{e}}$

$1.46^{\mathrm{g}}$

Benzoic acid

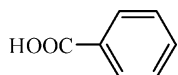

$\mathrm{p} K \mathrm{a}=4.20^{\mathrm{e}}$

$1.87^{\mathrm{h}}$ 
Table 1 (Continued)

Hame

${ }^{\text {a }}$ From reference [32].

${ }^{\mathrm{b}}$ From reference [33].

${ }^{\mathrm{c}}$ From reference [34].

${ }^{\mathrm{d}}$ From reference [35].

e From reference [36].

${ }^{\mathrm{f}}$ From reference [37].

g From reference [38].

${ }^{\mathrm{h}}$ From reference [39].

i The $\log K_{\text {ow }}$ value of 2,5-dihydroxybenzoic acid.

j The $\log K_{\text {ow }}$ value of 4-methoxyphenol.

${ }^{\mathrm{k}}$ From reference [40].

sium dihydrogen phosphate and $0.2 \mathrm{M}$ phosphoric acid) and water-ACN (50:50, $\mathrm{pH} 3.0$, adjusted by $0.2 \mathrm{M}$ potassium dihydrogen phosphate and $0.2 \mathrm{M}$ phosphoric acid) as the A and $\mathrm{B}$ solvent, respectively. For the other compounds the mobile phase consisted of water ( $\mathrm{pH} 3.0$, adjusted by $0.2 \mathrm{M}$ potassium dihydrogen phosphate and $0.2 \mathrm{M}$ phosphoric acid) and water-ACN $(20: 80)$ as the A and B solvent, respectively. The flow-rate for cephalosporin group was $1.0 \mathrm{~mL} \mathrm{~min}^{-1}$, and for other groups was $0.2 \mathrm{~mL} \mathrm{~min}^{-1}$. The linear gradient profile for cephalosporin group was B maintained $0 \%$ in the first $5 \mathrm{~min}, 17 \%$ in $5 \mathrm{~min}$, $100 \%$ in 6 min (held for $4 \mathrm{~min}$ ), after which the mobile phase was returned to the initial conditions in $5 \mathrm{~min}$. The gradient profile for sulfonamide group was B $12 \%$ in the first $10 \mathrm{~min}, 40 \%$ in $10 \mathrm{~min}$ (held for $5 \mathrm{~min}$ ), after which the mobile phase was returned to the initial conditions in $5 \mathrm{~min}$. And for phenol group, the gradient elution was performed by increasing linearly the percent of B from 5\% to 50\% in $10 \mathrm{~min}, 100 \%$ in $5 \mathrm{~min}$ (held for $2 \mathrm{~min}$ ), and then $\mathrm{B}$ was returned to the initial conditions in $5 \mathrm{~min}$. The wavelengths used to detect cephalosporin group, sulfonamide group and phenolic group was set at 270, 260 and $230 \mathrm{~nm}$, respectively.

\subsection{Solid-phase extraction cartridge}

The cartridge packed with $100 \mathrm{mg}$ of each sorbent was prepared in a $3 \mathrm{~mL}$ polypropylene syringe and the sorbent was retained by two polyethylene frits. The procedure with each cartridge for off-line enrichment was the same: the outlet tip of the cartridge was connected to a model DOA-P504-BN vacuum pump (GAST Manufacturing Inc., Mich., USA), and the inlet end of the cartridge was connected to a polytetrafluoroethylene (PTFE) suction tube whose other end was inserted into sample solution. Prior to use, the MWCNTs and SWCNTs were purified by $1 \mathrm{M}$ hydrochloric acid (sonicated about $2 \mathrm{~h}$ ) and washed with water till the sorbents were neutral.

\subsection{Water sample preparation}

A tap water sample was collected from our laboratory, and a well water sample came from the campus of China Agricultural University. A river water sample was collected from Jing Mi canal (Beijing). The collected water samples were filtered through $0.22 \mu \mathrm{m}$ nylon membranes (Shanghai Institute of Pharmaceutical Industry, Shanghai, China) immediately after sampling and were maintained in glass containers, then stored at a temperature of $4{ }^{\circ} \mathrm{C}$. Extraction of real water samples was carried out using MWCNTs packed cartridge. Five hundred millilitres of water sample or water sample spiked with analytes was passed through the cartridge for sample analysis or recovery test.

\section{Results and discussion}

\subsection{Desorption conditions}

It was well known that compounds adsorbed by GCBs sorbent were hard to desorb with conventional water miscible solvents 
Table 2

Desorption conditions for the investigated cephalosporin on carbonaceous sorbents

\begin{tabular}{|c|c|c|c|c|c|}
\hline Sorbent & Eluent & \multicolumn{4}{|c|}{ Recovery $(\%) \pm$ S.D. ${ }^{\mathrm{a}}(n=3)$} \\
\hline MWCNTs & $\mathrm{MeOH} /$ water $(9: 1)$ & $86.3 \pm 0.4$ & $94.7 \pm 2.3$ & $95.0 \pm 2.3$ & $92.2 \pm 1.2$ \\
\hline GCBs & $\mathrm{MeOH}$ & $90.1 \pm 2.9$ & $91.8 \pm 3.2$ & $92.7 \pm 3.1$ & $99.4 \pm 2.4$ \\
\hline
\end{tabular}

Eluent volume, $3 \mathrm{~mL}$; concentration of each analyte, $200 \mu \mathrm{g} \mathrm{L}^{-1}$; $\mathrm{pH}$ value of the solution, $\mathrm{pH} 6$.

a Standard deviation.

(such as $\mathrm{MeOH}$ or $\mathrm{ACN}$ ). Altenbach and Giger [41] reported that compounds with more than one amino group or hydroxyl group were more difficult to recover. Unfortunately, all the compounds tested in our study contained amino groups or hydroxyl group, and it was proved that satisfactory results could not be achieved with $\mathrm{MeOH}$ or ACN from GCBs sorbent. Similar difficulty was encountered to these analytes when they were extracted by CNTs sorbents. To obtain higher recoveries of the analytes, great efforts have been made to optimize the desorption conditions for three groups of compounds separately, including the composition of eluents, and volume of eluents.

\subsubsection{Composition of eluents}

In the present study, the cephalosporin group was the only one that could be eluted by $\mathrm{MeOH}$ or $\mathrm{ACN}$, or their mixture. The eluents for the cephalosporins to desorb from carbonaceous sorbents were listed in Table 2. As it was shown that, satisfactory recoveries for cephalosporins were obtained when extracted by CNTs or GCBs sorbents.

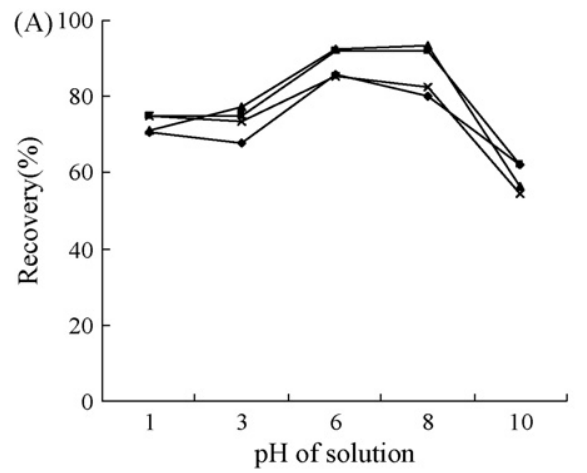

With regard to sulfonamide compounds, $\mathrm{MeOH}, \mathrm{ACN}$, and tetrahydrofuran were compared for the optimal selection. None of these solvents or their mixtures could produce better elution performance. It has been reported that, in the study of Altenbach and Giger [41], the aromatic sulfonates could quantitatively recover from GCBs adsorbent with the addition of ammonium acetate in eluent. So we selected several amino compounds (namely ammonium acetate, aminoacetic acid, or methylamine hydrochloride) as the additive of the eluent. It was turned out that remarkable increase in recoveries was achieved with ammonium acetate. And the effective eluent was made up of $30 \%$ of $0.3 \mathrm{M}$ ammonium acetate water solution and $70 \%$ of methanol.

The eluent of phenolic compounds was the same as that of sulfonamides. However, better recoveries were obtained only when the carbonaceous sorbents were pretreated with ascorbic acid. The pretreating procedure involved: washing the sorbents with $5 \mathrm{~mL}$ desorption solution, $5 \mathrm{~mL}$ methanol and $10 \mathrm{~mL}$ ascorbic acid $(0.03 \mathrm{M})$ in $0.1 \mathrm{M}$ hydrochloric acid and $10 \mathrm{~mL}$ pure water successively.

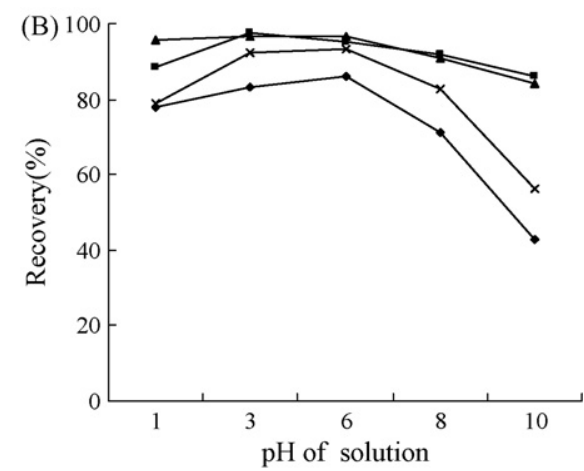

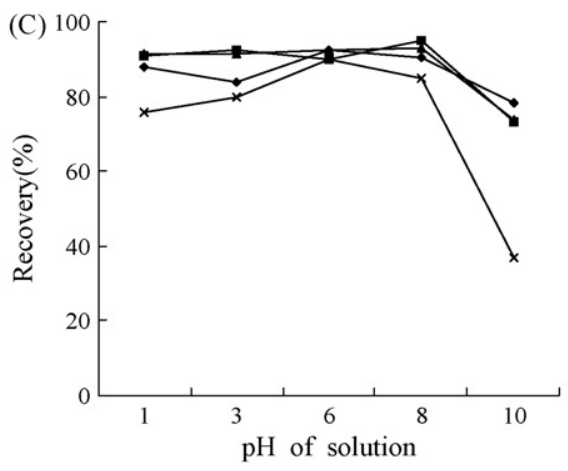

Fig. 1. Effect of solution pH on the recoveries of sulfonamide compounds extracted by carbonaceous adsorbents packed cartridges: (A) SWCNTs; (B) MWCNTs;

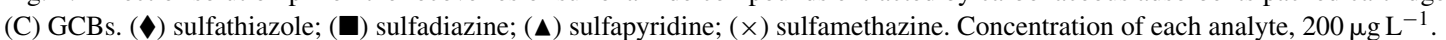




\subsubsection{Effect of eluent volume}

To find out the required volume of eluent to recover all the analytes from three carbonaceous sorbents packed cartridges, eluent volumes up to $6 \mathrm{~mL}$ were tested. It seemed that sulfonamide compounds needed larger volume of eluents ( $5 \mathrm{~mL}$ for each adsorbent) to get the best recoveries. For phenolic compounds, the smallest volume of eluents was demanded (3, 4 and $2 \mathrm{~mL}$ for MWCNTs, SWCNTs and GCBs adsorbent, respectively). Analytes enriched in SWCNTs sorbent need more eluents to desorb. In general, the volume of eluent used to recover the target analytes followed the order of: sulfonamides $>$ cephalosporins $>$ phenolic compounds.

\subsection{Effect of solution $\mathrm{pH}$}

The $\mathrm{pH}$ designed at the experiments was in the range of 1-10 to cover the protonated, unprotonated, partially deprotonated and deprotonated states of each polar compound. It was found out that the $\mathrm{pH}$ of sample solutions in the whole range had no significant influence on the extraction of the sulfonamides (see Fig. 1A-C). For the trapping of the cephalosporins and phenolic compounds, however, the situation was slightly different. As to the former, the recoveries of these compounds decreased at $\mathrm{pH}>8$ (see Fig. $2 \mathrm{~A}-\mathrm{C}$ ), meanwhile there were no cephalosporins detected in the alkaline percolate after their extraction by carbonaceous sorbents. It has been reported that cephalosporins were instable in alkaline medium $[42,43]$, therefore the lower recoveries at $\mathrm{pH}>8$ possibly resulted from their instability.

And for phenolic compounds, the dramatic decrease in recoveries (except guaiacol) was observed at $\mathrm{pH}>7$ (see Fig. 3A-C). The recoveries of hydroxyquinone and 1,3,5-trihydroxybenzene dropped to zero at $\mathrm{pH}>8$, meanwhile nearly none of the two analytes (except 1,3,5-trihydroxybenzene on MWCNTs) were found in percolate at $\mathrm{pH} 10$. Since the multi-hydroxyl phenols were easy to be oxidized in basic solution [44], we deduced that the loss was caused by the oxidation of these compounds at $\mathrm{pH}>8$. As to benzoic acid, no loss was found on GCBs sorbent at alkaline solution. But about $30 \%$ and $10 \%$ of decrease of recoveries at $\mathrm{pH} 10$ were obtained on MWCNTs and SWCNTs, respectively, yet the loss could be compensated by the amount present in percolate. Then it could be concluded that the ionization of benzoic acid had apparent effect on its retention on CNTs. With respect to 3,5dihydroxybenzoic acid and phenol, the recoveries decreased by $20-45 \%$ (MWCNTs $>$ SWCNTs $>$ GCBs) and $30-70 \%$ (SWCNTs $>$ GCBs $>$ MWCNTs) at $\mathrm{pH} 10$, respectively. However, the reduced recoveries could not be compensated by the amount present in percolate, indicating the combination effect of oxidation of hydroxyl groups and their ionizations. In summary, based on the experimental results above-mentioned, $\mathrm{pH} 6$ was chosen as the $\mathrm{pH}$ of the sample solutions.
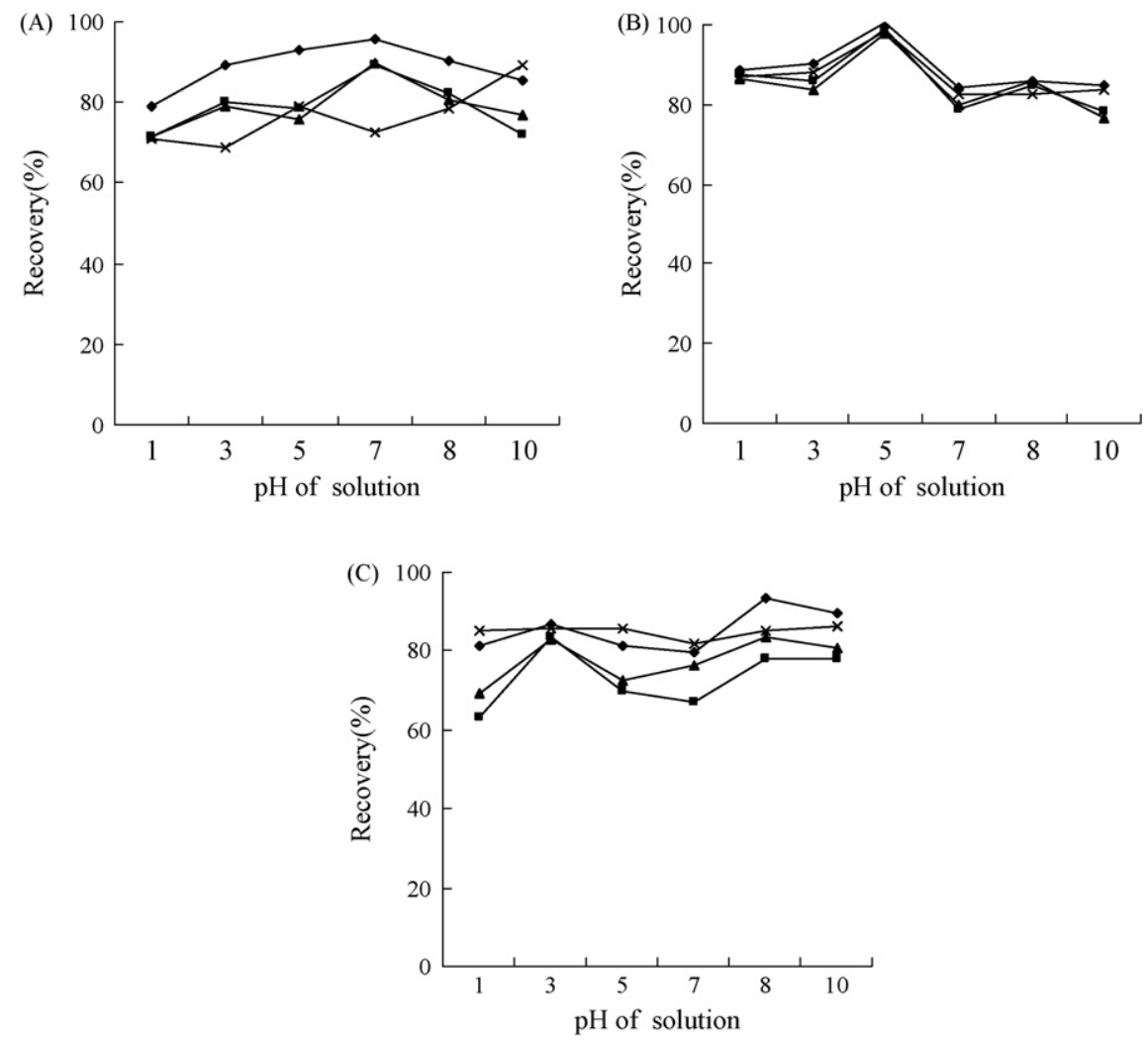

Fig. 2. Effect of solution $\mathrm{pH}$ on the recoveries of cephalosporin compounds extracted by carbonaceous adsorbents packed cartridges: (A) SWCNTs; (B) MWCNTs; (C) GCBs. ( ) cephaclor; $(\square)$ cephalexin; $(\mathbf{\Delta})$ cephradine; $(\times)$ cephalotin. Concentration of each analyte, $200 \mu \mathrm{g} \mathrm{L}^{-1}$. 

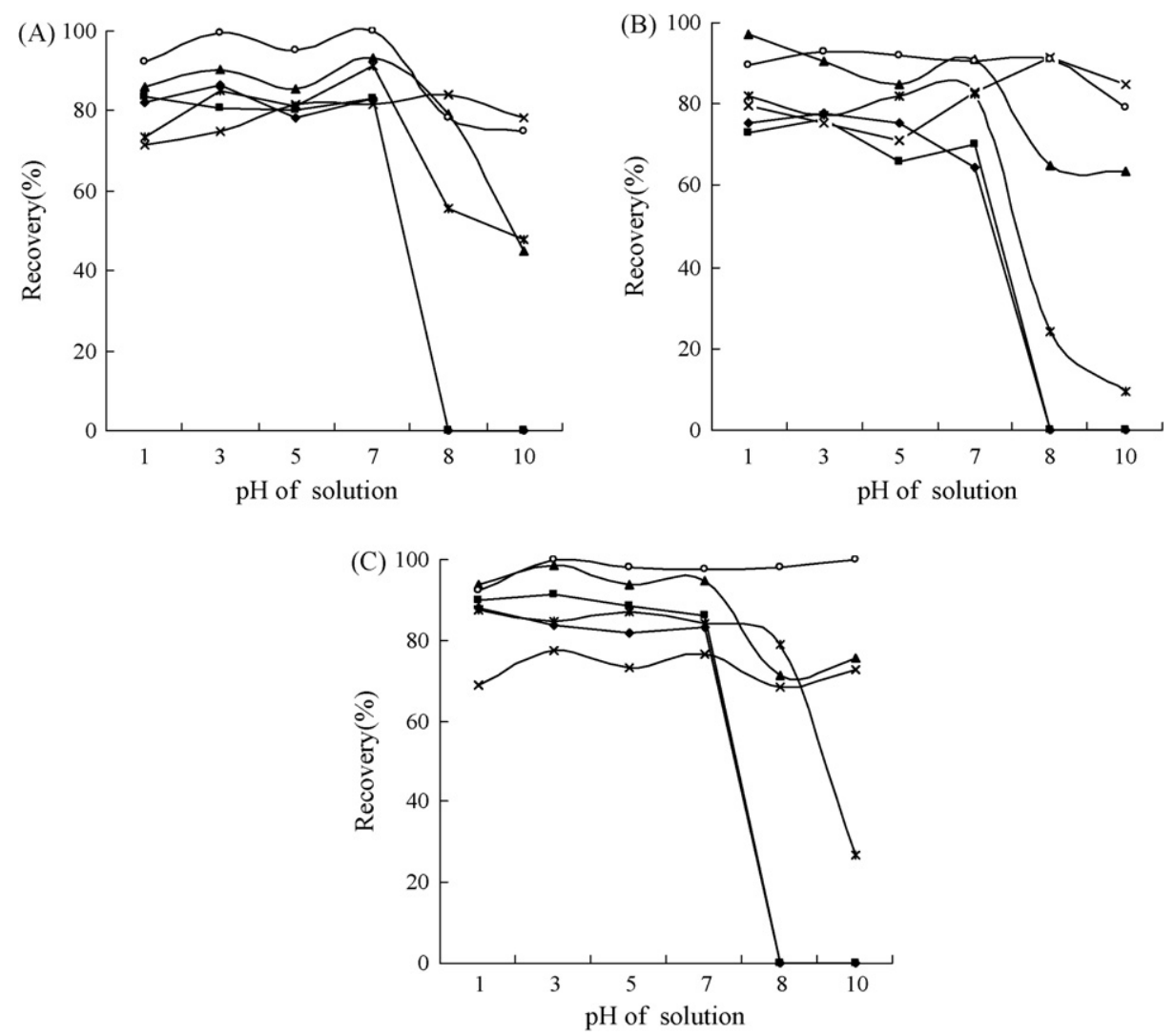

Fig. 3. Effect of solution $\mathrm{pH}$ on the recoveries of phenolic compounds extracted by carbonaceous adsorbents packed cartridges: (A) SWCNTs; (B) MWCNTs; (C) GCBs. ( ) 1,3,5-trihydroxybenzene; ( $\mathbf{\square})$ hydroxyquinone; (\) 3,5-dihydroxybenzoic acid; $(\times)$ guaiacol; $(*)$ phenol; $(\bigcirc)$, benzoic acid. Concentration of each analyte, $200 \mu \mathrm{g} \mathrm{L}^{-1}$.

\subsection{Analytical potential of CNTs for highly polar compounds}

Recoveries for the selected compounds were determined in the off-line SPE by preconcentrating different volumes of pure water spiked with a constant mass of $0.5 \mu \mathrm{g}$ of each polar analyte. The concentrations of the analytes depended on the preconcentrated volumes $(10-500 \mathrm{~mL})$ and were in the range of $1-50 \mathrm{ng} \mathrm{mL}^{-1}$. The analytes passed through the self-made cartridges packed with $100 \mathrm{mg}$ of CNTs and GCBs sorbents with a vacuum pump. The results were shown in Figs. 4-6. Though the amount of each sorbent used for SPE was very small, the recoveries of antibiotics showed little fluctuation with the increase of sample volume when they were trapped by CNTs. The recoveries of cephalosporins and sulfonamides were $>80 \%$ with the volume of $500 \mathrm{~mL}$. However, on GCBs sorbent, with the exception of sulfamethazine, it seemed that obvious loss happened for antibiotics when the sample volume was up to $500 \mathrm{~mL}$. With regard to phenolic compounds, significant loss of multi-hydroxyl phenol (hydroxyquinone and 1,3,5-trihydroxybenzene) and phenol was observed when the sample volume exceeded $10 \mathrm{~mL}$ or $25 \mathrm{~mL}$ both on CNTs or GCBs sorbents. However, no loss was observed for benzoic acid on each sorbent and for 3,5-dihydroxybenzoic acid on GCBs sorbent with a sample volume of $500 \mathrm{~mL}$; noticeable decrease of recovery occurred for 3,5-dihydroxybenzoic acid on CNTs only when the sample volume exceeded $100 \mathrm{~mL}$. In general, the antibiotics were more retained on CNTs sorbents than on GCBs, and extraction ability to these polar compound of three carbonaceous sorbents followed the order of MWCNTs $\geq$ SWCNTs $>$ GCBs. But for 3,5-dihydroxybenzoic acid, GCBs showed better enrichment ability than CNTs. As to multi-hydroxyl compounds (phenol included) relatively poorer retention were observed on each sorbent.

It is believed that strong interaction can take place between the graphitic ring structure of the carbon sorbents and compounds having benzene ring in their structures [11,15]. Since there were more benzoic rings, $\pi$ bonds or lone pairs of electrons in the structures of antibiotics than that of multihydroxyl phenols, stronger retention are obtained for antibiotics on carbon sorbents, leading to higher breakthrough volumes of antibiotics. Although possessing similar chemical structures, benzoic acid and 3,5-dihydroxybenzoic acid have higher breakthrough volumes on carbonaceous sorbents than other phenolic compounds indicating the different retention mechanism on carbon sorbents. Maybe there were anion-exchange sites (such as $-\mathrm{COOH},-\mathrm{OH}$ ) on the surface of the sorbents, and it would be helpful for the adsorption of benzoic acid and 3,5dihydroxybenzoic acid onto these sorbents when they were in their ionic form at $\mathrm{pH} 6$. The relatively lower breakthrough volumes for 3,5-dihydroxybenzoic acid on CNTs sorbents suggest fewer hydrophilic groups on CNTs surface than GCBs, which was also found in the research of Li et al. [45]. Probably, this is also the reason for the more loss of the two analytes on CNTs sorbents than on GCBs in alkaline solution. 

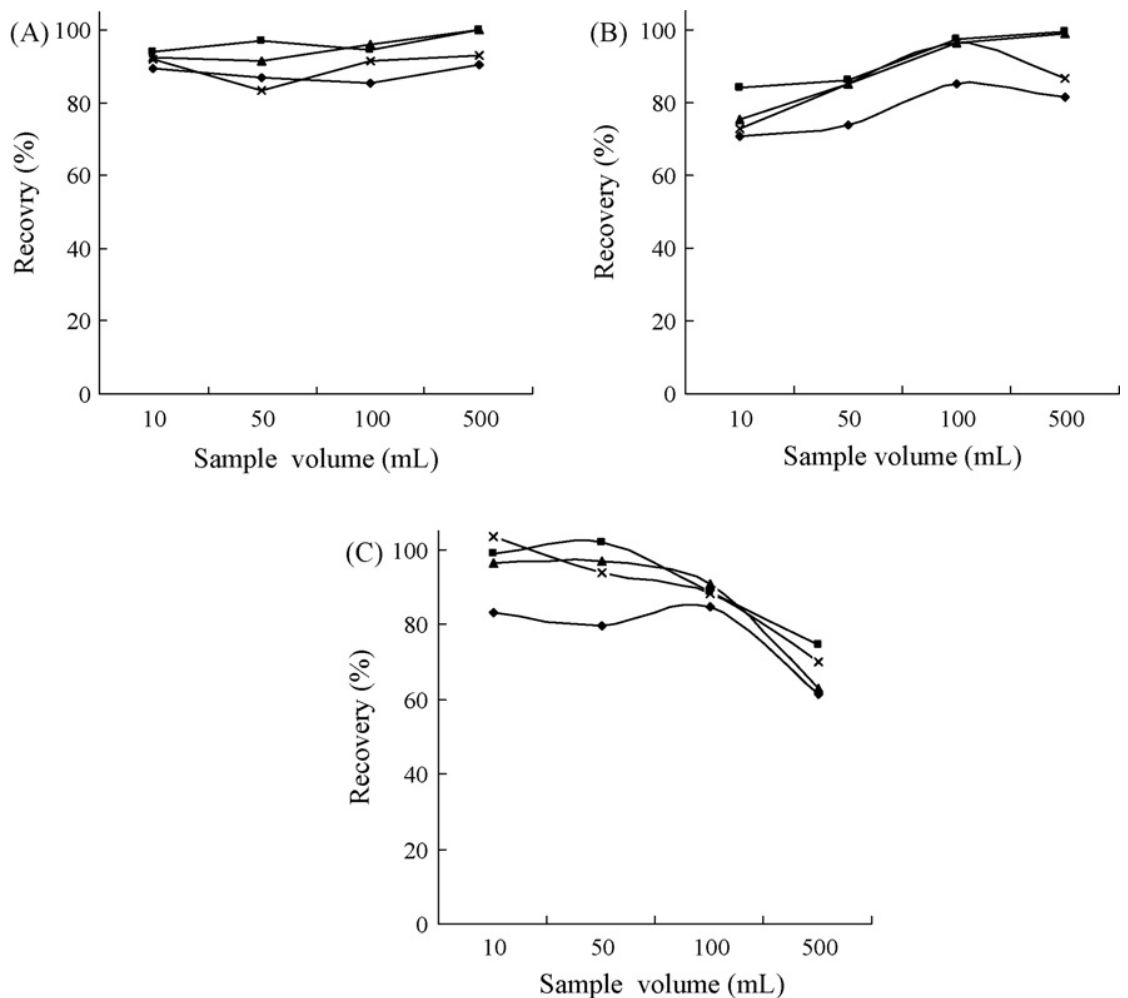

Fig. 4. Effect of sample volume on the recoveries of cephalosporin compounds extracted by the carbonaceous sorbents. The analytes amount is constant and equal to

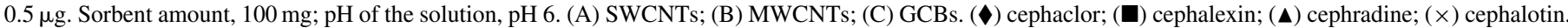
Standard deviation (S.D.) $(n=3)$ were lower than 10.
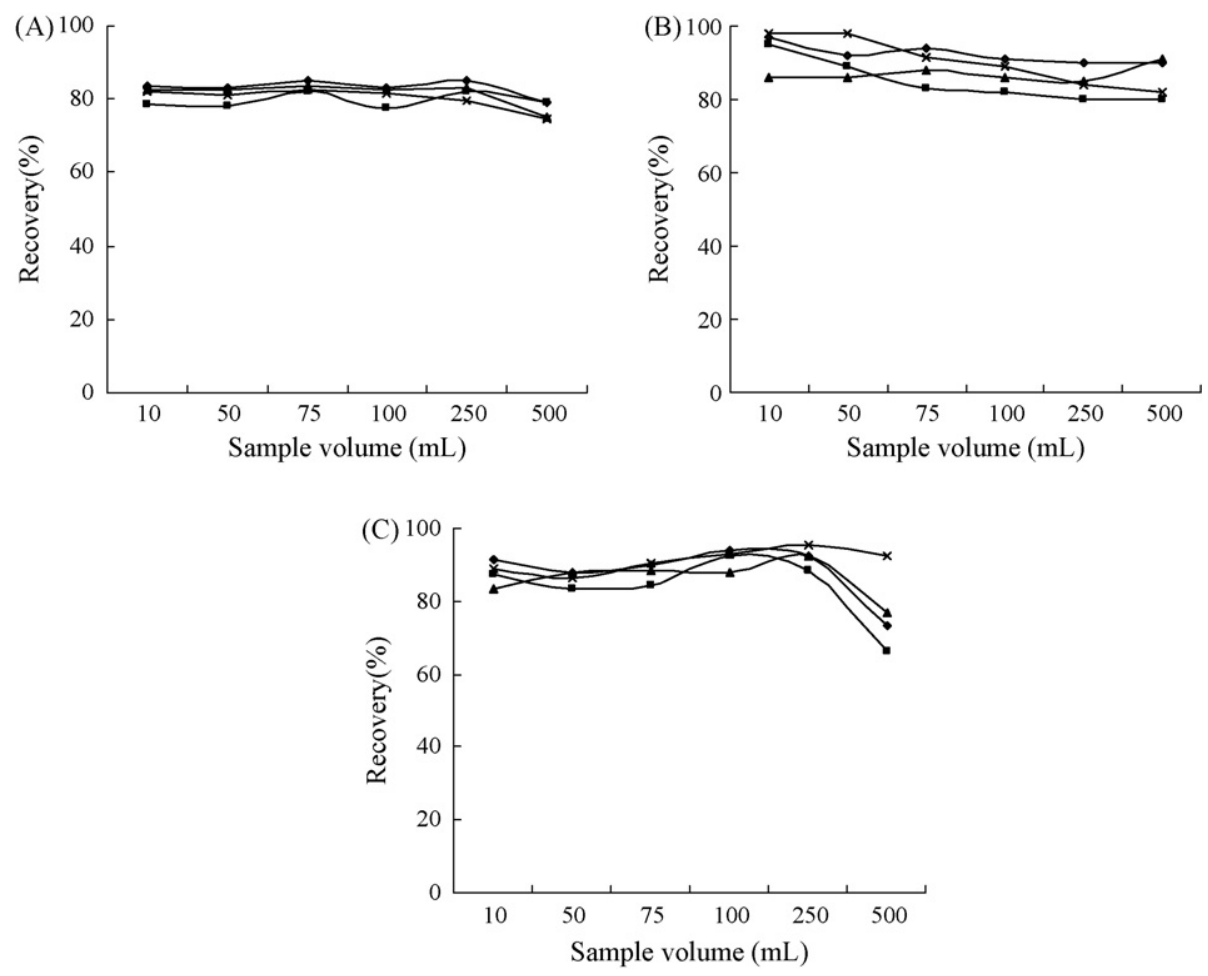

Fig. 5. Effect of sample volume on the recoveries of sulfonamide compounds extracted by the carbonaceous sorbents. The analytes amount is constant and equal

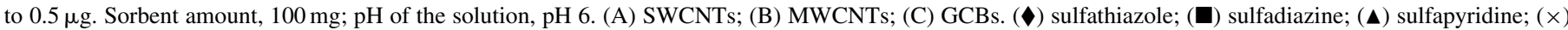
sulfamethazine. Standard deviation (S.D.) $(n=3)$ were lower than 10. 

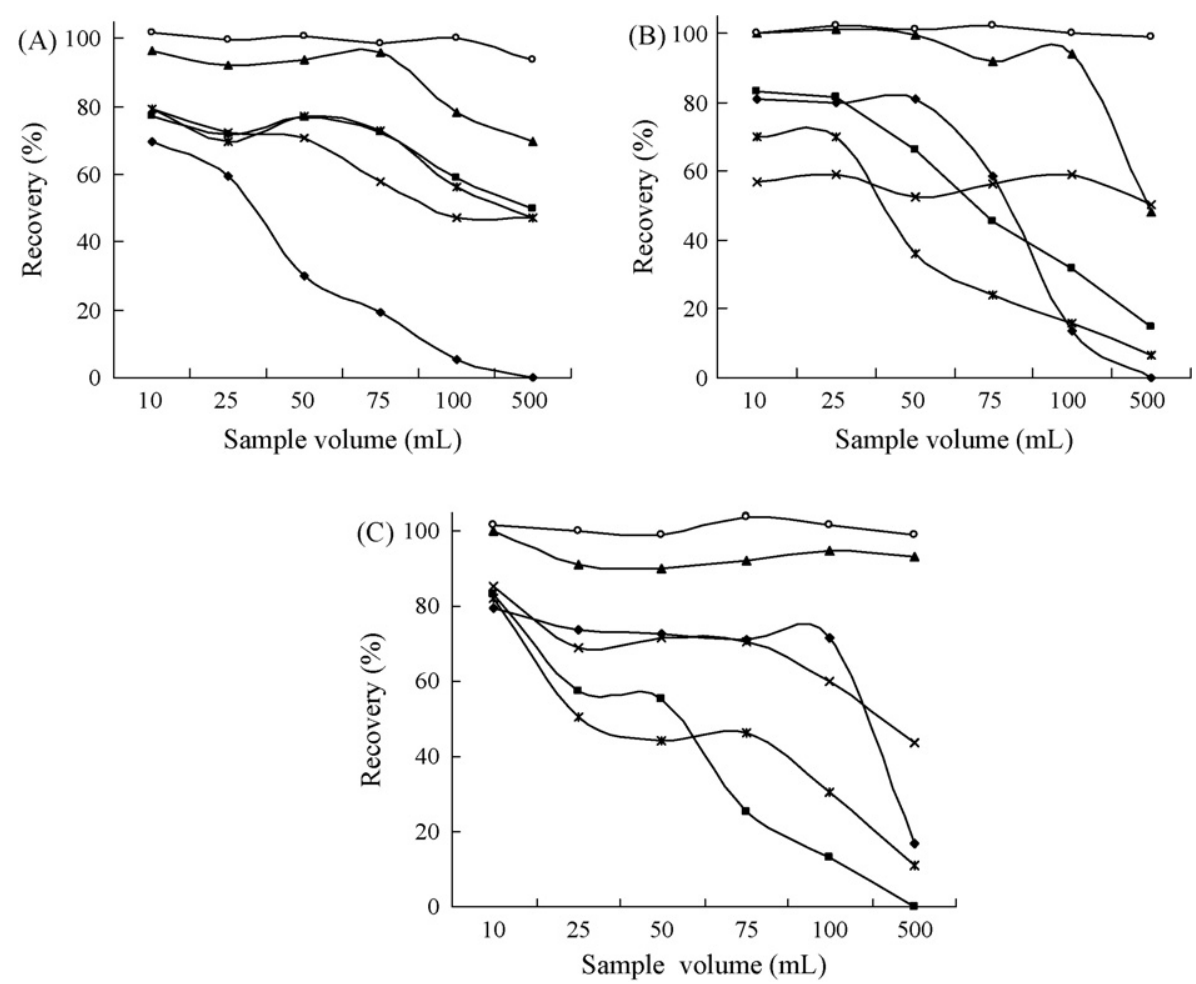

Fig. 6. Effect of sample volume on the recoveries of phenolic compounds extracted by the carbonaceous sorbents. The analytes amount is constant and equal to $0.5 \mu \mathrm{g}$. Sorbent amount, $100 \mathrm{mg}$; pH of the solution, pH 6. (A) SWCNTs; (B) MWCNTs; (C) GCBs. ( $)$ 1,3,5-trihydroxybenzene; (ם) hydroxyquinone; (ム) 3,5-dihydroxybenzoic acid; $(\times)$ guaiacol; $(*)$ phenol; $(\bigcirc)$ benzoic acid. Standard deviation $($ S.D. $)(n=3)$ were lower than 15.
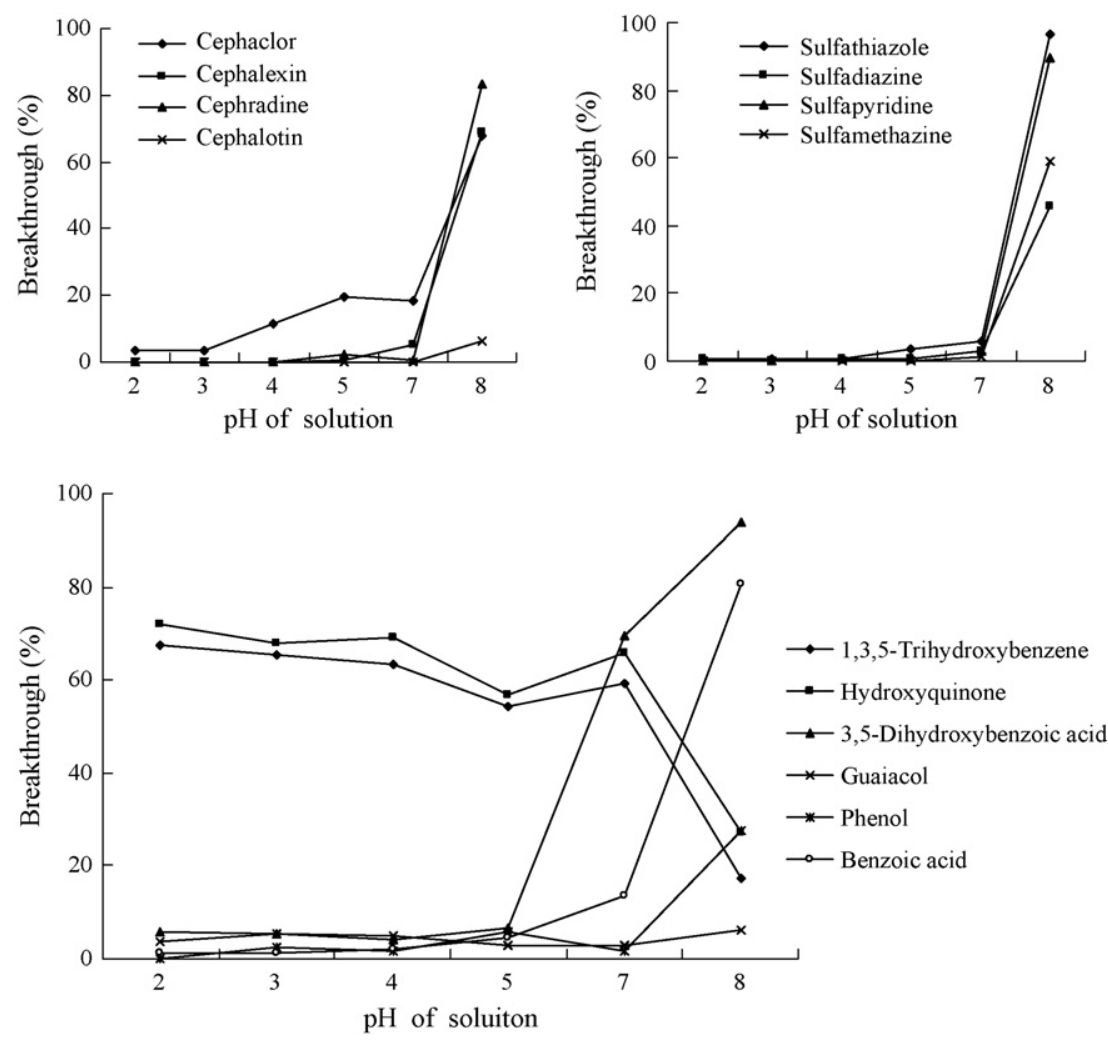

Fig. 7. Effect of solution $\mathrm{pH}$ on the recoveries of cephalosporin compounds, sulfonamides and phenolic compounds extracted by $\mathrm{C}_{18}$ packed cartridges. Concentration of each analyte, $2 \mu \mathrm{g} \mathrm{mL}^{-1}$ 

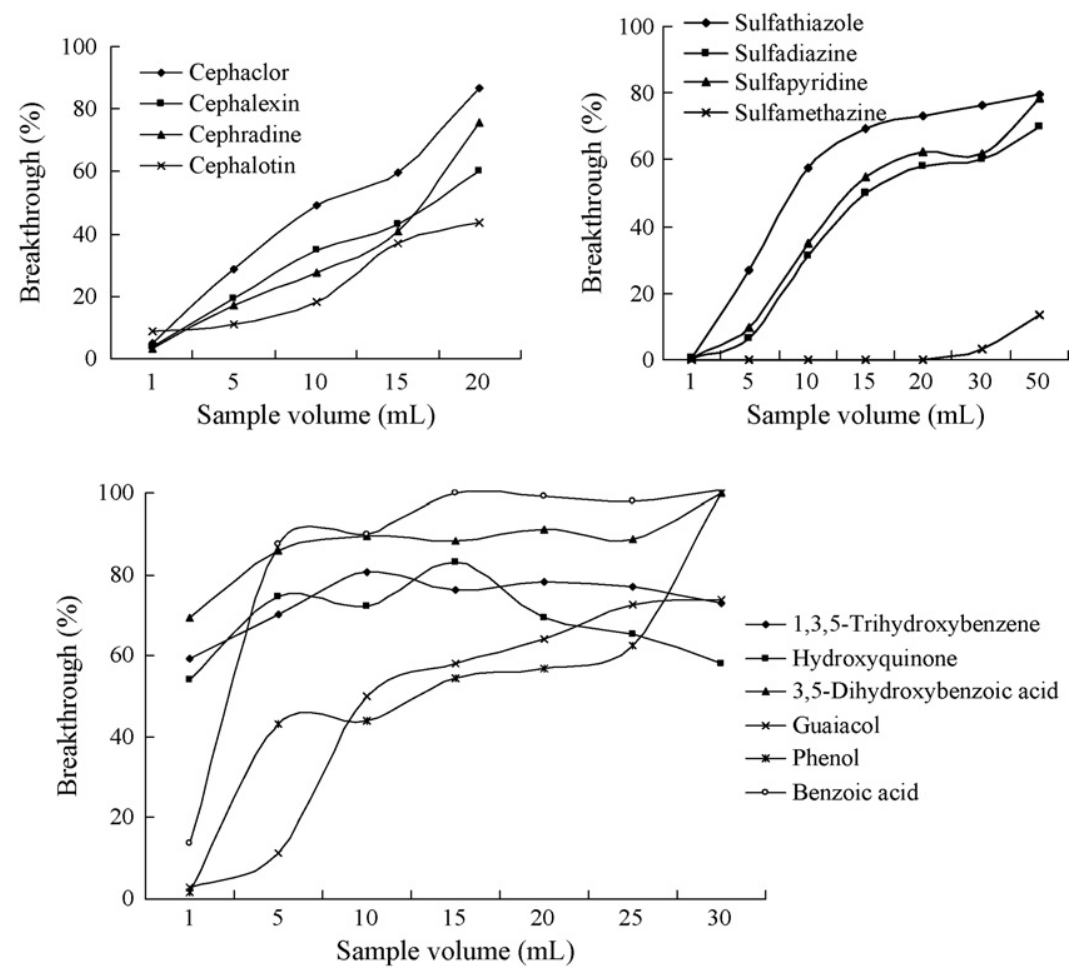

Fig. 8. Effect of sample volume on the recoveries of the cephalosporin compounds, sulfonamides and phenolic compounds extracted by $\mathrm{C}_{18}$ packed cartridges. The analytes amount is constant and equal to $0.5 \mu \mathrm{g}$. Sorbent amount, $100 \mathrm{mg}$; $\mathrm{pH}$ of the solution, $\mathrm{pH} 6$.

\subsection{Comparison study}

For comparative studies, the most popular used solid-phase extraction sorbent $\mathrm{C}_{18}$ was selected. Following the same experimental procedure and conditions as the carbonaceous sorbents packed cartridges, the effect of solution $\mathrm{pH}$ on recoveries, and retention behavior of each analyte were investigated. Because of the high breakthrough of these analytes on $\mathrm{C}_{18}$ silica, the concentration in percolate of each analyte was determined instead of their recoveries. Unlike the situation on carbonaceous sorbents, the adsorption of these polar compounds on $\mathrm{C}_{18}$ silica sorbent shifted drastically with the variation of solution $\mathrm{pH}$ in the range of 2-8. Generally, the amount of most of these polar compounds detected in percolate was increasing with the rising of $\mathrm{pH}$ (shown in Fig. 7). For phenolic compounds, it should be mentioned that the decrease of the 1,3,5-trihydroxybenzene and hydroxyquinone in percolate at solution $\mathrm{pH} 7-8$ resulted from their susceptibility to oxygen. Not surprisingly, the breakthrough volumes for most of these analytes on $\mathrm{C}_{18}$ sorbent were below $1 \mathrm{~mL}$ indicating the poor retention abilities for polar compounds (see Fig. 8). Therefore, the $\mathrm{C}_{18}$ was not the suitable candidate to extract trace level of highly polar compounds from environmental samples.

\subsection{Analytical performance and sample analyses}

Sulfonamides are among the most widely used antimicrobials in human and veterinary medicine. It has been reported that sulfonamides have high potential to resist degradation, and can be transferred into aquatic environment [46]. Several of sulfonamides (such as sulfamethazine and sulfamethoxazole) have been detected in wastewater, surface water, and even groundwater samples [47-56], which has led to great concerns about the potential for the development of antimicrobial resistance amongst microorganisms. Consequently sulfonamides were used as model compounds in this section. According to the results obtained above, the MWCNTs sorbent showed satisfactory extraction ability for sulfonamides than the other sorbents, thus the MWCNTs packed cartridge was used to analyze real water samples. To ensure all the analytes were collected into the eluent completely, $10 \mathrm{~mL}$ eluent was used to desorb the components. The extracts were concentrated on a rotary evaporator to about $0.5 \mathrm{~mL}$, and then diluted to $1.0 \mathrm{~mL}$ by methanol. Under the optimized conditions, the analytical performance of the proposed method was assessed with $500 \mathrm{~mL}$ of spiked pure water samples and the results were presented in Table 3 . It can be seen that the present method has high sensitivity and a wide linear range.

The proposed method had been applied to the analysis of some environmental water samples. None of the analytes were found in the samples. To evaluate the accuracy of this method, $500 \mathrm{~mL}$ of environmental water samples spiked with the mixture of analytes at 0.50 or $5.00 \mathrm{ng} \mathrm{mL}^{-1}$ were preconcentrated and analyzed by this system, and the recoveries were given in Table 4. It was shown that the recoveries for sulfapyridine and sulfamethazine in all the samples were rather satisfactory and in the range of $85-102 \%$, but the recoveries of sulfathiazole and sulfadiazine were relatively lower in all samples, and the spiked recoveries for sulfathiazole and sulfadiazine were in the range of $55-79 \%$ and $72-92 \%$, respectively. Compared with the recov- 
Table 3

Linear range, calibration equations, correlation coefficients and detection limits of the analytical system

\begin{tabular}{|c|c|c|c|c|}
\hline Compound & Linear range $\left(\mathrm{ng} \mathrm{mL}^{-1}\right)$ & Calibration equations & $R^{2}$ & Detection limit $\left(\mathrm{ng} \mathrm{L}^{-1}\right)(s / n=3)$ \\
\hline Sulfathiazole & $0.10-20.00$ & $y=1.1679 x-0.0122$ & 0.9999 & 27 \\
\hline Sulfadiazine & $0.10-20.00$ & $y=0.8963 x-0.0969$ & 0.9992 & 34 \\
\hline Sulfapyridine & $0.10-20.00$ & $y=1.1309 x+0.0219$ & 0.9999 & 38 \\
\hline Sulfamethazine & $0.10-20.00$ & $y=1.0982 x-0.0575$ & 0.9998 & 29 \\
\hline
\end{tabular}

Table 4

Recoveries of real water samples spiked with the sulfonamide compounds

\begin{tabular}{|c|c|c|c|c|c|}
\hline \multirow[t]{2}{*}{ Water samples } & \multirow[t]{2}{*}{ Spiked (ng mL ${ }^{-1}$ ) } & \multicolumn{4}{|c|}{ Confidence limit of recovery $(\%)(n=3)^{\mathrm{a}}$} \\
\hline & & Sulfathiazole & Sulfadiazine & Sulfapyridine & Sulfamethazine \\
\hline \multirow[t]{2}{*}{ Tap water } & 0.5 & $79 \pm 4$ & $72 \pm 3$ & $92 \pm 2$ & $92 \pm 6$ \\
\hline & 5.0 & $72 \pm 9$ & $85 \pm 1$ & $85 \pm 7$ & $86 \pm 4$ \\
\hline \multirow[t]{2}{*}{ Well water } & 0.5 & $88 \pm 3$ & $92 \pm 2$ & $95 \pm 3$ & $100 \pm 4$ \\
\hline & 5.0 & $66 \pm 4$ & $80 \pm 2$ & $102 \pm 1$ & $101 \pm 7$ \\
\hline \multirow[t]{2}{*}{ Jingmi canal } & 0.5 & $55 \pm 6$ & $87 \pm 8$ & $95 \pm 6$ & $91 \pm 8$ \\
\hline & 5.0 & $56 \pm 5$ & $75 \pm 0.1$ & $87 \pm 6$ & $89 \pm 7$ \\
\hline
\end{tabular}

${ }^{\text {a }} p=90 \%$.

eries of sulfonamides obtained in other studies, the extraction efficiency of MWCNTs adsorbent was superior to MCX adsorbents (mixed mode strong cation exchange resin) (recoveries of sulfadiazine and sulfathiazole were less than 50\%) [48] or $\mathrm{C}_{2} / \mathrm{EVN}+$ adsorbents (recoveries of sulfadiazine and sulfathiazole were no more than 20\%) [50,56]. Furthermore, with the exception of sulfathiazole, the recoveries of sulfonamides were comparable with those extracted by hydrophilic-lipophilic balance (HLB) copolymer wildly used adsorbent for antibiotics [51-56]. As sulfamethazine was one of the most commonly used sulfonamides mainly for veterinary applications [51], it could be frequently detected in environmental aqueous samples. The recovery of sulfamethazine was satisfactory in all the samples, which indicated that this analyte can be quantitatively detected using this developed method. Interestingly, after the treatment of MWCNTs cartridge, there were little interferences for the determination of sulfonamides when analyzed by HPLC, and the chromatograms of the river water sample and its spiked solution were shown in Fig. 9. In a word, the MWCNTs adsorbents can

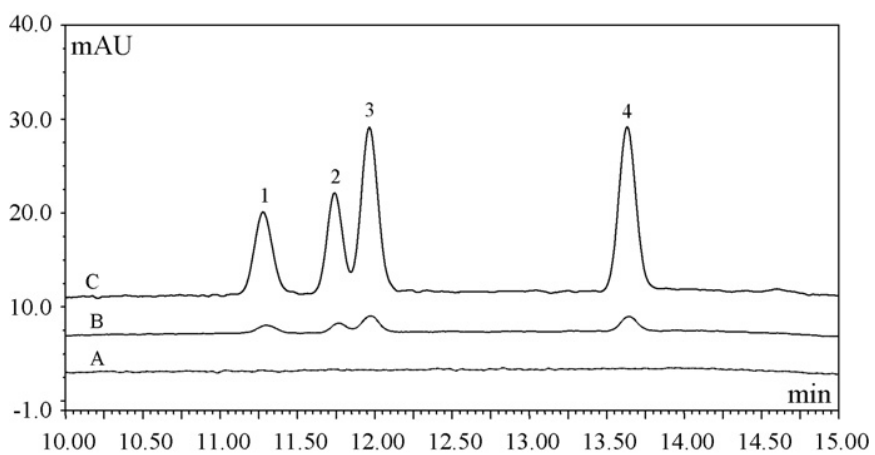

Fig. 9. Solid-phase extraction/LC-UV chromatograms of river water sample preconcentrated by MWCNTs packed cartridge. (A) river water sample; (B) river water sample spiked with $0.50 \mathrm{ng} \mathrm{mL}^{-1}$ of each compound; and (C) river water sample spiked with $5.00 \mathrm{ng} \mathrm{mL}^{-1}$ of each compound. (1) sulfathiazole; (2) sulfadiazine; (3) sulfapyridine; (4) sulfamethazine. Volume of water sample solution, $500 \mathrm{~mL}$; See text for other experimental conditions. be regarded as a new kind of potent adsorbent for the detection of sulfonamides from environmental samples.

\section{Conclusions}

In this paper, the applicability of the CNTs as packing sorbents for SPE of highly polar analytes was investigated. The compounds selected (including cephalosporins, sulfonamides, and phenolic compounds) bearing multi-functional groups such as $-\mathrm{NH}_{2},-\mathrm{OH}$, or $-\mathrm{COOH}$ in their structure were difficult to desorb when extracted by CNTs or GCBs. Great efforts had been made to optimize the desorption conditions, and satisfactory recoveries of the highly polar analytes were obtained which were in the range of $80-100 \%$. The recoveries of most of the analytes decreased at solution $\mathrm{pH}>8$. Comparative studies showed that the CNTs were much superior to $\mathrm{C}_{18}$ for the extraction of the highly polar analytes. In general, the antibiotics were more retained on CNTs sorbents than on GCBs sorbent. As to multi-hydroxyl compounds (phenol included) relatively poorer retention were observed on each sorbent, but for 3,5-dihydroxybenzoic acid, GCBs showed better enrichment abilities than CNTs. Finally MWCNTs packed cartridge was selected to preconcentrate sulfonamide compounds from several real water samples. It is turned out that MWCNTs have great potential for SPE of highly polar compounds.

\section{Acknowledgments}

This work was jointly supported by the National Natural Science Foundation of China (20475060) and Major Research Program of Chinese Academy of Sciences (KZCX3-SW-432).

\section{References}

[1] M.C. Hennion, J. Chromatogr. A 856 (1999) 3.

[2] M.C. Hennion, J. Chromatogr. A 885 (2000) 73. 
[3] N. Fontanals, M. Galià, R.M. Marcé, F. Borrull, J. Chromatogr. A 1030 (2004) 63.

[4] N. Fontanals, M. Galià, P.A.G. Cormack, R.M. Marcé, D.C. Sherrington, F. Borrull, J. Chromatogr. A 1075 (2005) 51.

[5] N. Fontanals, R.M. Marcé, F. Borrull, Trends Anal. Chem. 24 (2005) 394.

[6] K. Bielica-Daszkiewicz, A. Voelkel, M. Szejner, J. Osypiuk, Chemosphere 62 (2006) 892.

[7] A.A. D'Archivio, M. Fanelli, P. Mazzeo, F. Ruggieri, Talanta 71 (2007) 25.

[8] S. Iijima, Physica B 323 (2002) 1.

[9] A. Merkoçi, M. Pumera, X. Llopis, B. Pérea, M.D. Valle, S. Alegret, Trends Anal. Chem. 24 (2005) 826.

[10] S. Iijima, Nature (Lond.) 354 (1991) 56.

[11] Y. Li, Y. Chen, R. Xiang, D. Ciuparu, L.D. Pfefferle, C. Horváth, J. Wilkins, Anal. Chem. 77 (2005) 1398.

[12] R.Q. Long, R.T. Yang, J. Am. Chem. Soc. 123 (2001) 2058.

[13] C.S. Lu, Y.L. Chung, K.F. Chang, Water Res. 39 (2005) 1183.

[14] H. Yan, A. Gong, H.S. He, J. Zhou, Y.X. Wei, L. Lv, Chemosphere 62 (2006) 142.

[15] X.J. Peng, Y.H. Li, Z.K. Luan, Z.C. Di, H.Y. Wang, B.H. Tian, Z.P. Jia, Chem. Phys. Lett. 376 (2003) 154.

[16] Z.C. Chen, L.S. Zhang, Y.W. Tang, Z.J. Jia, Appl. Surf. Sci. 252 (2006) 2933.

[17] Q.X. Zhou, W.D. Wang, J.P. Xiao, Anal. Chim. Acta 559 (2006) 200.

[18] Q.X. Zhou, Y.J. Ding, J.P. Xiao, Chromatographia 65 (2006) 25.

[19] Y.Q. Cai, G.B. Jiang, J.F. Liu, Q.X. Zhou, Anal. Chim. Acta 494 (2003) 149.

[20] Y.Q. Cai, Y.E. Cai, S.F. Mou, Y.Q. Lu, J. Chromatogr. A 1081 (2005) 245.

[21] Y.Q. Cai, G.B. Jiang, J.F. Liu, Q.X. Zhou, Anal. Chem. 75 (2003) 2517.

[22] B. Suárez, B.M. Simonet, S. Cárdenas, M. Valcárcel, J. Chromatogr. A (2007), doi:10.1016/j.chroma. 2007.01.092

[23] J.X. Wang, D.Q. Jiang, Z.Y. Gu, X.P. Yan, J. Chromatogr. A 1137 (2006)

[24] C. Saridara, S. Mitra, Anal. Chem. 77 (2005) 7094

[25] Q.L. Li, D.X. Yuan, Q.M. Lin, J. Chromatogr. A 1026 (2004) 283.

[26] Q.L. Li, D.X. Yuan, J. Chromatogr. A 1003 (2003) 203.

[27] Q.X. Zhou, J.P. Xiao, W.D. Wang, G.G. Liu, Q.Z. Shi, J.H. Wang, Talanta 28 (2006) 1309.

[28] Q.X. Zhou, J.P. Xiao, W.D. Wang, Anal. Sci. 23 (2007) 189.
[29] C. Basheer, A.A. Alnedhary, B.S.M. Rao, S. Valliyaveettil, H.K. Lee, Anal. Chem. 78 (2006) 2853

[30] C.S. Pan, S.Y. Xu, H.F. Zou, Z. Guo, Y. Zhang, B.C. Guo, J. Am. Soc. Mass Spectrom. 16 (2005) 263

[31] M. Biesaga, K. Pyrzynska, J. Sep. Sci. 29 (2006) 2241.

[32] http://www.medicinescomplete.com/mc/clarke/current/index.htm.

[33] Y. Mrestani, M. Janich, H.H. Rüttinger, R.H.H. Neubert, J. Chromatogr. A 873 (2000) 237.

[34] F. Martínez, A. Gómez, J. Phys. Org. Chem. 15 (2002) 874.

[35] A.L. Batt, D.D. Snow, D.S. Aga, Chemosphere 64 (2006) 1963.

[36] www.zirchrom.com/organic.htm.

[37] W.H. Douglas, J. Dent. Res. 57 (1978) 800.

[38] http://www.ilo.org/encyclopaedia.htm (ICSC).

[39] G.H. Lu, S.Q. Jiang, Y.H. Zhao, Chem. J. Internet 3 (2001) 34.

[40] http://ntp.niehs.nip.gov/index.cfs.

[41] B. Altebbach, W. Giger, Anal. Chem. 67 (1995) 2325.

[42] S. Thiele-Bruhn, J. Plant Nutr. Soil. Sci. 166 (2003) 145.

[43] M.E. Abdel-Hamid, Il Farmaco 53 (1998) 132.

[44] A.D. Corcia, A. Bellioni, M.D. Madbouly, S. Marchese, J. Chromatogr. A 733 (1996) 383

[45] Y.H. Li, Y.Q. Zhu, Y.M. Zhao, D.H. Wu, Z.K. Luan, Diamond Relat. Mater. 15 (2006) 90

[46] N.T. Malintan, M.A. Mohd, J. Chromatogr. A 1127 (2006) 154.

[47] E.R. Campagnolo, K.R. Johnson, A. Karpati, C.S. Rubin, D.W. Kolpin, M.T. Meyer, J.E. Esteban, R.W. Currier, K. Smith, K.M. Thu, M. McGeehin, Sci. Total Environ. 299 (2002) 89.

[48] V.K. Balakrishnan, K.A. Terry, J. Toito, J. Chromatogr. A 1131 (2006) 1.

[49] C. Hartig, T. Storm, M. Jekel, J. Chromatogr. A 854 (1999) 163.

[50] R. Lindberg, P.Å. Jarnheimer, B. Olsen, M. Johansson, M. Tysklind, Chemosphere 57 (2004) 1479.

[51] A. Göbel, C.S. McArdell, M.J.-F. Suter, W. Giger, Anal. Chem. 76 (2004) 4756.

[52] X.S. Miao, F. Bishay, M. Chen, C.D. Metcalfe, Environ. Sci. Technol. 38 (2004) 3533

[53] J.E. Renew, C.H. Huang, J. Chromatogr. A. 1042 (2004) 113.

[54] S.W. Yang, J.M. Cha, K. Carlson, J. Chromatogr. A 1097 (2005) 40.

[55] M.E. Lindsey, M. Meyer, E.M. Thurman, Anal. Chem. 73 (2001) 4640.

[56] F. Sacher, F.T. Lange, H.-J. Brauch, I. Blankenhorn, J. Chromatogr. A 938 (2001) 199 